\title{
Development of Simulator for a Material Handling System Analysis of Assembly Line
}

\author{
Dae S. Chang and Sang C. Park
}

\begin{abstract}
T Presented in this paper is the development of the simulator for In-plant MHS (Material Handling System) analysis of the assembly line. The MHS congestion on the assembly line prevents feeders and vehicles from traveling freely on the assembly line. Accordingly, congestion in a MHS can reduce the performance of the system. Although there have been many existing studies for reducing congestion in the system, they are difficult to apply to a work-site directly, because most of them have been studied in mathematical approaches. An intuitive way is to apply a satisfactory result of simulator to analyze current system and new system. The proposed simulator to perform the simulation uses simulation model, simulation engine and Feeder manager. The MHS congestion analysis simulation model consists of five nodes: the vehicle node, the buffer node, the work node, the source node and the sink node. The simulation engine has an event calendar to store events, and the engine repeatedly calls the events on the event calendar. The feeding manager which performs a role of route and schedule of feeders is the main idea of an MHS analysis simulator. The implemented simulator can be utilized to analyze the efficiency of MHS and working balance among feeders.
\end{abstract}

Index Terms-Material handling system, simulator, assembly line.

\section{INTRODUCTION}

In recent years, home appliance companies have shortened the lifecycle of home appliances due to technology advances and various needs of customers. To survive and prosper in the modern manufacturing era, a home appliance company should be capable of adapting shortened lifecycle of home appliances in a continuously changing marketplace, as shown in Fig. 1. Home appliance companies should develop new products and be able to produce new products at the same time to meet the needs of customers. However, it is difficult to develop and produce new products at the same time. One of the main obstacles in the production of new products comes from the different design and weight of new products, because if the weight and design of products are changed, factory layout design may have to be changed.

The factory layout design can be defined as rationally allocating resources (workshop, machines, storages, workers etc.) to the plant site, based on production process and production standard. A general factory layout problem is concerned with the physical organization and allocation of a production system [1]. There are mathematical approaches of the factory layout design for automatically generating factory

Manuscript received February 27, 2014; revised April 18, 2014.

The authors are with the Department of Industrial Engineering, Ajou University, Republic of Korea (e-mail:DaesChang@gmail.com, scpark@ajou.ac.krF). layout [2]. However, the mathematical approaches have the limitation of the incidental and accidental situations. In addition, the designers have difficulties to use mathematical approaches in work-site due to the low flexibility and a lot of constraints. Pandit and Palekar [3] use simulation to study response time in warehouse design. Uttapol and Sakapoj [4] use simulation tool 'Arena' to generate plant layout alternative.However, the designers have difficulties in using existing simulation approaches, because they are not experts of simulation. Currently, most factory layout design approach in the actual work-site is using the designers' know-how. The approach in actual work-site may involve both advantages and disadvantages of the past factory layout. The factory layout designer should apply the advantages of the past production line without the disadvantages to the new factory layout, but it is difficult to find and compensate the disadvantages in the past factory layout. Accordingly, we developed production system design simulator and found out interconnectivity between factory layout design and material handling system congestion during the improvement period of simulator.

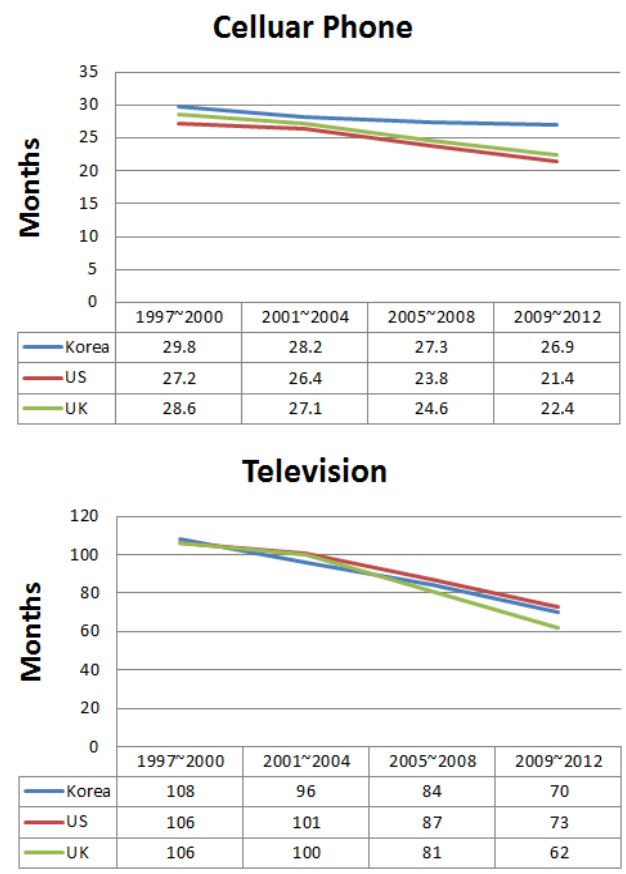

Fig. 1. Development of lifecycle of home appliance.

In-plant MHS must supply products parts to the assembly line in appropriate time. If the MHS works well, assembly line doesn't have supply-starved situations. As mentioned before, the assembly line can be changed by product changes. In addition, the assembly line can be shortened because of modularity and process improvement. In that case, the 
in-plant MHS has to be redesigned to supply the part well according to the changes on an assembly line [5]. However, schedule and route of feeders (part suppliers in the plant) in the plant may not operate as intended by the congestion on the assembly line, because, schedule and route problem of feeders are associated with the MHS congestion on the assembly line. The MHS congestion on the assembly line prevents feeders and vehicles from traveling freely on the assembly line. Consequently the MHS congestion needs to be analyzed [6]. In this paper, we developed a simulator for analysis of the MHS congestion, because existing mathematical approaches are difficult to predict unexpected situations. Also, we can design alternative schedule and route of feeders and predict unexpected situations using simulator.

Companies that produce products during designing production lines consider the three details as follows. The first is the placement of the equipment. The placement of the equipment on an assembly line is usually placed by the assembly operation sequence of the products. The second is the MHS design and the selection problem of the aisle or path. From the point of view in the MHS, the equipment on the assembly line are destinations provided parts and obstacles which feeders have to avoid. The MHS aisle or path can be varied by placement of equipment on the assembly line previously. The third is the MHS design. The MHS design is an action which plans vehicle schedule and parts assigned to be fed into the assembly line. The production line such as home appliances assembly line mostly has a high frequency of changes. The assembly line takes high cost of the changes if production line use automated MHS. For this reason, companies are flexible in responding to operate the assembly line based on the results of the assembly line changes [7]-[9]. Previous research gathers the actual data of current situation and uses algorithms to solve a series of problems for designing automated MHS, or calculates the shortest path to generate MHS design alternatives on an assembly line, or carries out the genetic algorithm to generate the MHS alternatives [10]-[12]. Past studies focus on automatically generating MHS design alternatives. This paper has focused on identifying patterns of In-plant logistics, congestion analysis of the assembly line, the route of the vehicles and the feeders. This paper has two major objectives: (1) to propose a proper structure for MHS simulator and (2) to implement the simulator for analyzing the MHS congestion by simulating the alternative factory layout. The simulator area of the proposed methodology includes all types of flow manufacturing systems supplied by feeders, such as refrigerator, television, cellular phone, air conditioner, washing machine and automotive assembly lines. The overall structure of the paper is as follows. Section II addresses the overall approach to simulate the factory layout for analyzing MHS congestion. Section III details the construction of simulators for analyzing the MHS congestion. Section IV shows some illustrative examples, along with a result of the implemented simulator. Finally, concluding remarks are given in Section V.

\section{ApPROACH TO THE CONGESTION ANALYSIS OF THE MHS IN PLANT}

When we construct a new assembly line or change the existing assembly lines, a lot of factors must be considered. Mostly the assembly line such as home appliance production line has following factors to be considered; 1) assembly sequence of the product; 2) a number of operations; 3) part supply; 4) the job allocation problem of workers; 5) working balance of each worker; 6) production flow speed. To consider the aforementioned factors, it is necessary to utilize simulation techniques for support of alternative generation. By simulating factory layout alternatives, it is possible to find optimized MHS in various ways and recognize hidden MHS operation alternatives more intuitively. Although MHS simulation can be a very powerful tool for the operation verification of a production system, the simulation techniques may not be familiar to the designers, therefore, the simulation techniques itself can be a major obstacle. The parts supply problem in considerations of design for production system has many alternative solutions. However, it is hard to evaluate whether the alternatives are feasible or infeasible because there are lots of considerations such as the number of the feeders and operating vehicles in the parts supply problem.

\begin{tabular}{|c|c|c|}
\hline & & Description of the role of each model \\
\hline \multirow{5}{*}{$\begin{array}{l}\text { Simulation } \\
\text { Model }\end{array}$} & $\begin{array}{l}\text { Source } \\
\text { Node }\end{array}$ & Release new main parts into the assembly line. \\
\hline & $\begin{array}{l}\text { Buffer } \\
\text { Node }\end{array}$ & Keep the main part until next 'Work' is idle. \\
\hline & $\begin{array}{l}\text { Work } \\
\text { Node }\end{array}$ & Seize the main part for working time. \\
\hline & $\begin{array}{l}\text { Vehicle } \\
\text { Node }\end{array}$ & Supply sub parts to 'Buffer' nodes. \\
\hline & $\begin{array}{l}\text { Sink } \\
\text { Node }\end{array}$ & Count and ship the finished products. \\
\hline \multirow{2}{*}{$\begin{array}{l}\text { Data } \\
\text { Model }\end{array}$} & $\begin{array}{l}\text { Road } \\
\text { Data }\end{array}$ & $\begin{array}{l}\text { Contain the set of poly line as road in the } \\
\text { plant. }\end{array}$ \\
\hline & $\begin{array}{l}\text { Depot } \\
\text { Data }\end{array}$ & $\begin{array}{l}\text { Contain the location data of depots in the } \\
\text { plant. }\end{array}$ \\
\hline
\end{tabular}

The proposed simulator was designed so that can support easy and rapid modeling capabilities to the designer to design a MHS by using topology information and limited geometry data for each operation. The geometry data containsthe position of the depot, position of the buffer in the flow line, aisle in plant, position of vehicles and so on. The topology information contains a sequential relationshipbetween the operations and connection relationship between part, workers, feeder and depot [13]. Also, we analyze the production system and models for the analysis of the MHS could be classified into two types of model. The first model is a simulation model which consists of five types of nodes: 'Source' node, 'Work' node, 'Buffer' node, 'Sink' node and 'Vehicle' node (see Table I). The 'Source' nodes release new main parts into assembly line by inter-arrival time. The 'Work' nodes seize the main part and release the main part after working time. In storing the main and subpart, the 'Buffer' node passes the parts to the next 'Work' node when the 'Work' node is idle state. The 'Vehicle' node supplies subparts which are necessary for each 'Buffer' node. The 'Sink' nodes count and ship the finished product in the assembly line to out of the plant. The second model is a data 
model which contains 'Road' data and 'Depot' data. The 'Road' data consists of a set of direct polylines to represent aisle on the plant. The 'Depot' data contains the position and size of the depot, each depot has different types of part. In addition, such data as tact time of the assembly line, capacity of the buffer and the speed of vehicle should be input by the designer.

To build each node, this paper employs Zeigler's DEVS (Discrete Event Systems Specifications) formalism (Zeigler 1984, Kim 1994) [14], [15], is short for discrete event system specification. It dictates the modular and hierarchical formalism to model and analyze general systems that can be represented as discrete event systems. These mantics of the formalism are highly compatible with object-oriented specifications for simulation models. We use the atomic model of the DEVS formalism to represent the behavior of a device model. Formally, an atomic model $M$ is specified by a 7-tuple:

$$
\mathrm{AM}=\left\langle X, Y, S, \delta_{\text {int }}, \delta_{\text {ext }}, \lambda, t_{a}\right\rangle
$$

$X$ : input events set

$Y$ : output events set

$S$ : states set

$\delta_{\text {int }} S \rightarrow S$ : internal transition function

$\delta_{\text {ext }} Q \times X \rightarrow \mathrm{S}$ : external transition function

$Q=\left\{(s, e) \mid s \in S, 0 \leq e \leq t_{a}(s)\right\}$ : total state of AM

$\lambda S \rightarrow \mathrm{Y}$ : output function

$t_{a} S \rightarrow R_{\infty}^{+}$: time advance function

The four elements in the 7-tuple, namely $\delta_{\text {int }}, \delta_{\text {ext }}, \lambda$ and $t_{a}$, are called the characteristic functions of an atomic model. The atomic model of the DEVS formalism can be considered as a timed-FSA (finite state automata), and it is suitable for describing the behavior of the modeling nodes. Once the modeling nodes are obtained, it becomes possible to model the MHS simulation model and simulate the MHS as a discrete event system easily. Therefore, we modeled the nodes(Source, Buffer, Work, Vehicle and Sink are described details later) for supporting to design the MHS. So, we could design the new MHS by the result that we can analyze the congestion and the pattern of feeder operation during the simulation, which inputted real data of the plant.

\section{THE MHS SiMULATION CONSTRUCTION}

The MHS simulation model contains simulation model and data model. The simulation model contains the five nodes which have to be modeled by using the DEVS formalism in advance, and the data model contains set of real data for performing the MHS simulation. Fig. 2 illustrates how the five nodes are modeled by using DEVS. This is necessary to model the five node functions. As mentioned above, each node has a specific role, details are as follows: As shown in Fig. 2, a source node generates main parts at interval of 'GEN TIME' until 'SIM STOP' input-event entered, and main parts can be various types of part depend on the finished product. The types of main part have to be input by the user when the user models a source node. A sink node counts out of the number of finished products when products arrive sink node until condition 1 is satisfied or the simulation time passes 'SIM END TIME'. Also, the sink node takes finished product out of the assembly line. A buffer node has to communicate with a work node. Once a 'Part-In' signal firing comes in, a buffer node increases the number of the parts in the buffer node. And, a buffer node transfers a part of the next work node after transit time if state of work node is 'WAIT', the buffer node is changed state 'FREE' to 'BUSY' by itself at the same time. A work node seizes received part for working time, and then passes the part next node. At the same time, a work node changes state of the connected buffer node 'BUSY' to 'FREE'. A vehicle node has important role which delivers parts from depot to buffer nodes and induces congestion on the assembly line. A vehicle node has important role which delivers parts from depot to buffer nodes and induces congestion on the assembly line. The vehicle node has the travel time, the loading time, the unloading time and route. The travel time can be got by calculating vehicle speed and distance between departure and destination. The vehicle speed, the loading time and the unloading time have to be input by user. The route is composed of the depot, buffer nodes and the road. Also, routes have to be defined by the user, and each route belongs to singular or plural vehicles.

The construction of the MHS Simulation is based on construction of the discrete event simulation. A simulation engine has an event calendar which contains events. The event contains target node, time and input type. All nodes in simulation model can reserve event to the event calendar. Once a new event is reserved, the scheduled events in event calendar are sorted in time order. And then, the simulation engine takes the first event out of event calendar. The simulation engine changes the simulation time to the specific time that the event has to be occurred and fires the first event. Once the event is fired, four nodes (except a vehicle node) which related with the event schedule a new event in event calendar. The event related to a vehicle node can be scheduled by a feed manager. The feed manager is monitoring the WIP (work in process) in each buffer during the simulation. When the WIP in the buffers is less than a standard amount which user input, the feed manager finds out the idle vehicle which has to supply the part of the buffer node, and it makes vehicle node schedule a new event.

The vehicle node has several routes which contain a depot, a road and buffers, and supplies the parts to buffers in each route. All routes are designed by user. If there is no idle vehicle to supply the parts to empty buffers when the feed manager is monitoring, buffers can be starved. Once a buffer node is starved, the buffer node counts the number of starvation, and the number will be reported to the user after the MHS simulation. The user can determine the number of vehicles in MHS with considering the reported number as a simulation result. Also, the vehicle node counts the number of each route used (TF). The number can be used for route allocation and the congestion analysis of MHS. Each route can calculate total travel distance (TD) to supply the parts to the buffers using positions of depot, buffers and a road. We can calculate the congestion level using mentioned data. The congestion level equation can be described as follows:

\section{A. Notation}

$C L=$ congestion level

$S R_{I}=$ ratio of sharing an aisle among routes at route $i$ 
$T I_{i}=$ transport intensity of the route $i$,

$T D_{i}=$ total travel distance of the route $i$

$T F_{i}=$ transport frequency of the route $i$,

$S L_{i}=$ Length of shared aisle at route $i$

$N=$ number of routes in the MHS,

$M=$ number of shared routes in the MHS
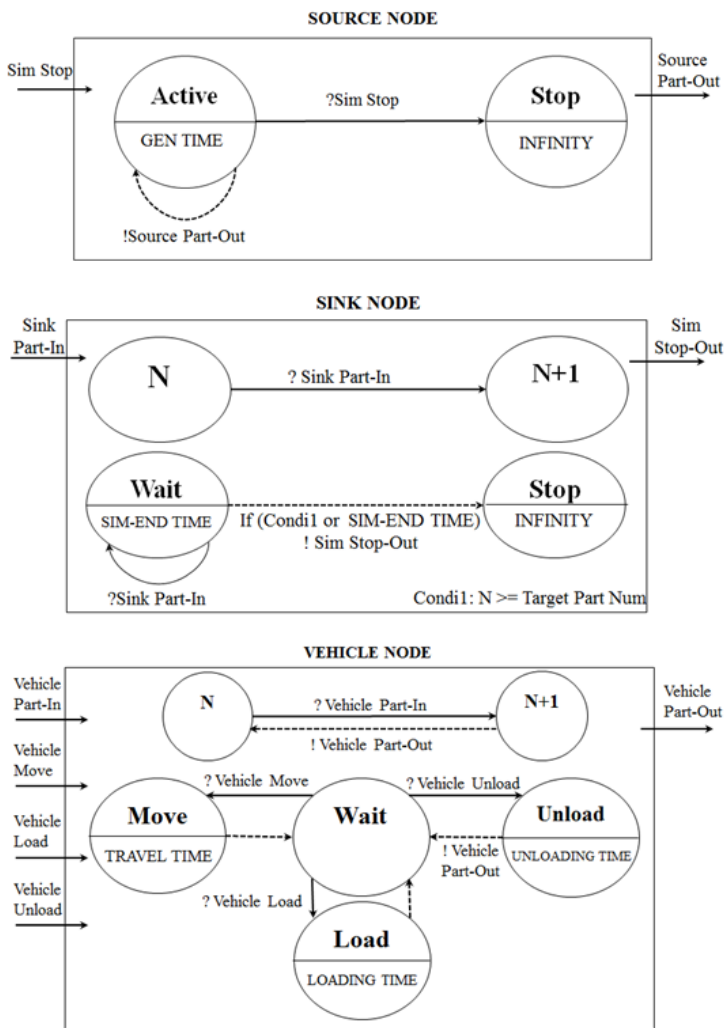

Fig. 2. Atomic models: a Source Node, a Work Node, a Sink Node, a Buffer Node and a Vehicle Node.

Source Node

$X=\{$ Source Part-In $\}$

$Y=\{$ Source Part-Out $\}$

$S=\{$ Active, Stop $\}$

$\delta_{\text {int }}($ Active $)=$ Active

$\delta_{\text {ext }}$ (Active, Sim Stop) $=$ Stop

$\lambda($ Active $)=$ Source Part-Out

$t_{a}($ Active $)=$ GEN TIME $($ User input $)$

Sink Node

$X=\{$ Sink Part-In $\}$

$Y=\{$ Sim Stop-Out $\}$

$S=\{$ Wait, Stop $\}$

$\delta_{\text {int }}$ (Wait) $=$ Stop

$\delta_{\text {ext }}($ Wait, Sink Part-In $)=$ Wait

$\lambda$ (Wait) $=$ Sim Stop-Out

$t_{a}$ (Wait) $=$ SIM-END TIME(User input $)$
$X=\{$ Buffer Part-In, Work Ready $\}$

$Y=\{$ Buffer Part-Out $\}$

$S=\{$ Free, Busy $\}$

$\delta_{\text {int }}($ Free $)=$ Busy

$\delta_{\text {ext }}($ Busy, Work Ready $)=$ Free

$\lambda($ Free $)=$ Buffer Part-Out

$t_{a}($ Active $)=$ TRANSIT TIME(User input $)$

Work Node

$X=\{$ Work Part-In $\}$

$Y=\{$ Work Part-Out, Work Ready-Out $\}$

$\delta_{\text {int }}$ (Busy) $=$ Wait

$\delta_{\text {ext }}($ Wait, Work Part-In $)=$ Busy

$\lambda$ (Busy) $=$ Work Part-Out

$\lambda$ (Busy) $=$ Work Ready-Out

$t_{a}$ (Busy) $=$ WORKING TIME(User input $)$
$S=\{$ Busy, Wait $\}$

\section{B. Congestion Level Equation}

$$
\begin{gathered}
C L=T I i \times \sum_{i=1}^{N} S R i \\
T I_{i}=T D_{i} \times T F_{i} \\
S R_{i}=S L_{i} / T D_{i} \times M
\end{gathered}
$$
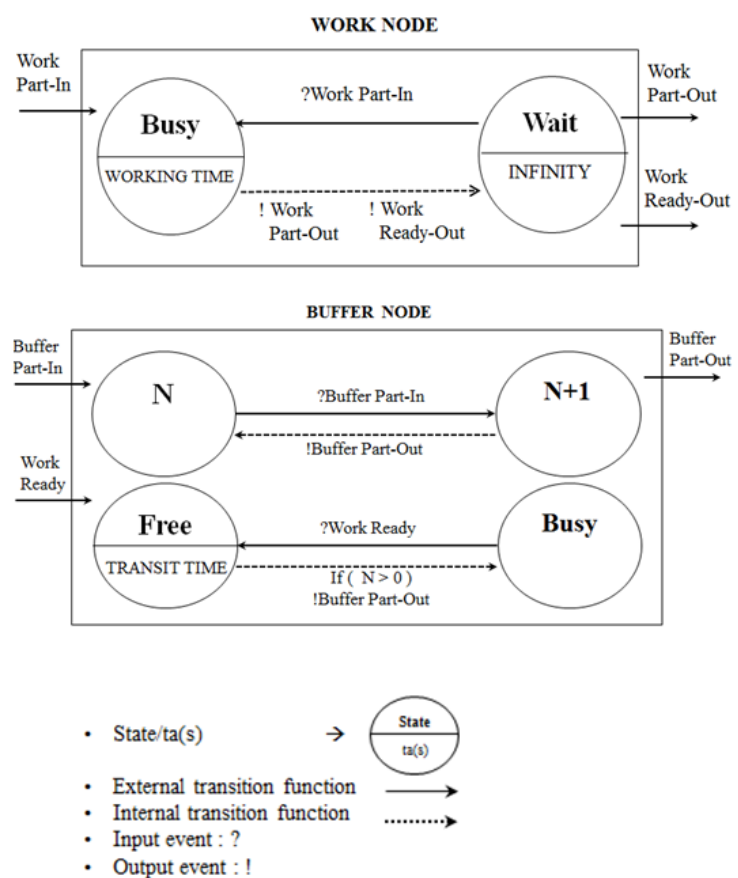

- Input event : ?

Output event :

\section{Vehicle Node}

$X=\{$ Vehicle Part-In, Vehicle Move, Vehicle Load, Vehicle Unload $\}$

$Y=\{$ Vehicle Part-Out $\}$

$S=\{$ Wait, Move, Unload, Load $\}$

$\delta_{\text {int }}($ Move $)=$ Wait, $\delta_{\text {int }}($ Load $)=$ Wait, $\delta_{\text {int }}($ Unload $)=$ Wait

$\delta_{\text {ext }}($ Wait, Vehicle Move $)=$ Move, $\delta_{\text {ext }}($ Wait, Vehicle Load $)=$ Load,

$\delta_{\text {ext }}($ Wait, Vehicle Unload $)=$ Unload

$\lambda($ Unload $)=$ Vehicle Part-Out

$t_{a}($ Move $)=$ TRAVEL TIME(User input $), t_{a}($ Load $)=$ LOADING TIME(User input $)$

$t_{a}($ Unload $)=$ UNLOADING TIME(User input $)$

Notation for nodes atomic model depicted in Fig. 2. 


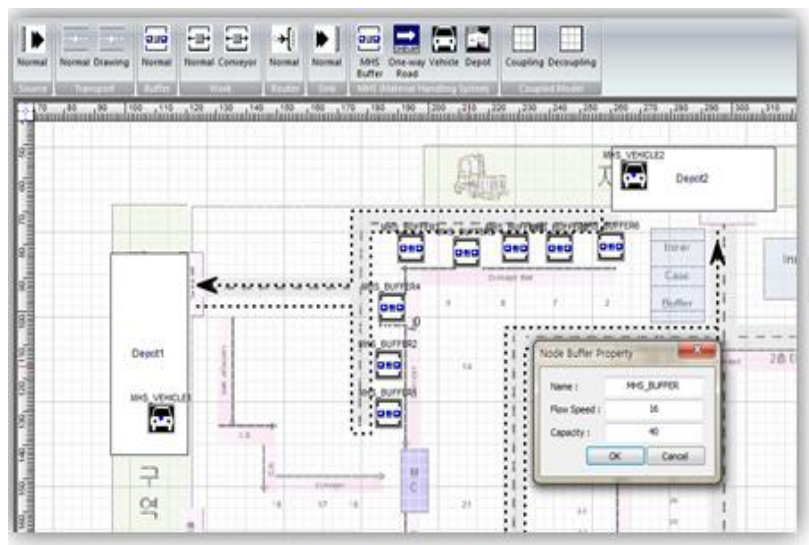

(1) MHS Modeling

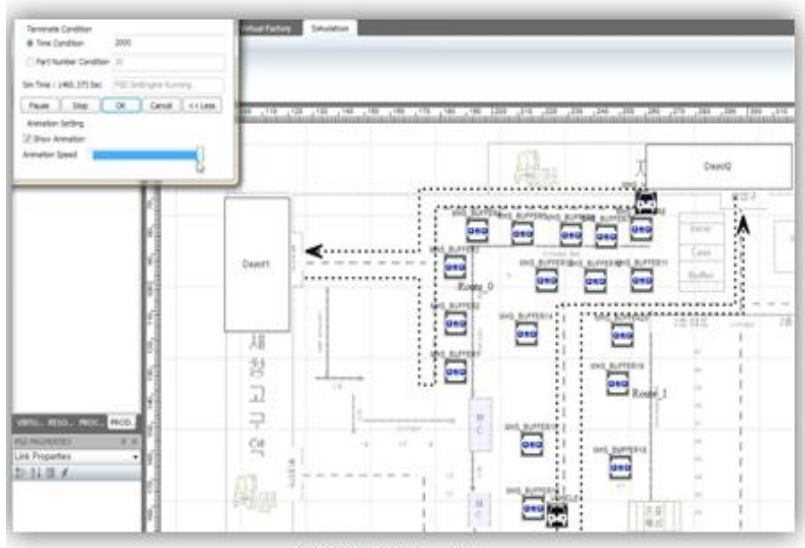

(3) MHS Simulation

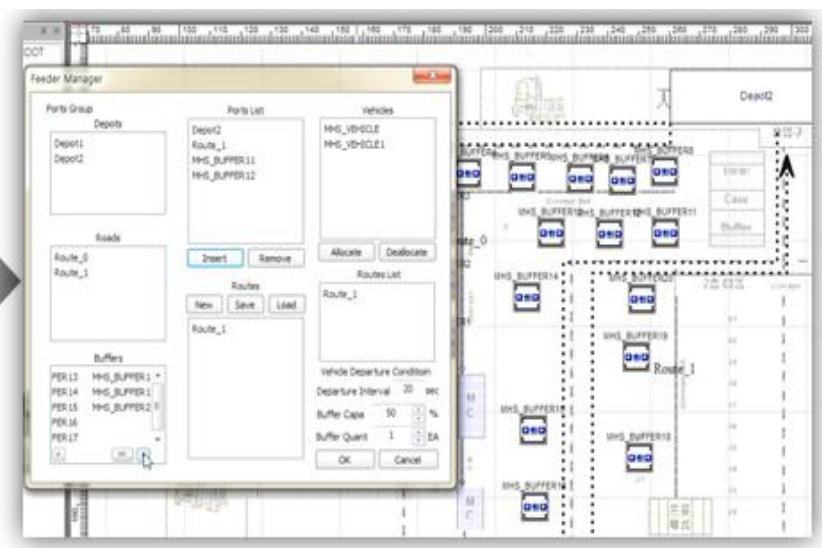

(2) Route design

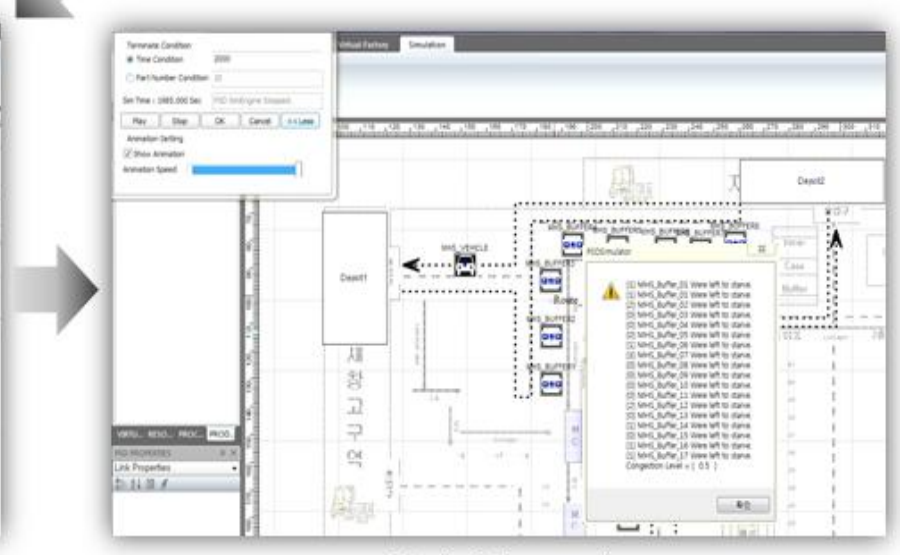

(4) Simulation Result

Fig. 3. The demonstration of the MHS simulation program.

\section{DEVELOPMENT OF MHS SIMULATOR}

The proposed MHS simulator has been implemented and tested with refrigerator assembly line to prove its usefulness. For the simulation of the MHS model, this paper employs Zeigler's DEVS formalism. We used $\mathrm{C}++$ language in a Visual Studio environment and E-XD++ library for the graphical user interface (GUI). The implemented program supports drag and drop method and simple user interface (UI) to model the MHS to the user.

The demonstration of the MHS simulator shown in Fig. 3 is a part of the refrigerator assembly line with two vehicles and two routes to supply seventeen buffers. The refrigerator assembly line is the longest and most complex assembly line in five electronics assembly lines which are refrigerator, mobile phone, TV, air conditioner and washer. So, it has to be improved as short as the other assembly line continuously. We simplified the actual refrigerator factory floor plan and used as a background of simulation visualization. Fig. 3(1) shows the MHS modeling user interface. The users can model the five nodes using five buttons on the toolbar and input the essential details such as the vehicle speed and buffer capacity through $\mathrm{C}++$ dialog. Fig. 3(2) shows the route design user interface. The user can design the routes and allocate route to vehicles. After route design, we can simulate the MHS model and confirm the movement of the vehicles through animation during the simulation, as shown in Fig. 3(3). Our final goal is to analyze a congestion of the MHS on assembly line for helping the MHS design. Therefore, users can easily design the MHS, without making efforts for ancillary tasks such as scripting and coding. We can confirm the congestion analysis result by executing program with an MHS model, as shown in Fig. 3(4).

\section{CONCLUSION}

This paper presents an MHS simulation program for the analysis congestion of an MHS in an assembly line. For the implementation of the proposed MHS simulation program, the paper employs DEVSIM++ which is an object-oriented simulation language based on Zeigler's DEVS formalism. For the factory layout design decision, it is associated with the MHS in the assembly line. MHS simulation can be a very powerful tool for the production system design. But, the designers may not be familiar with simulation techniques. Therefore, the simulation tool should support an easy way to the designers. The proposed MHS simulation program contains five types of modeling nodes: the vehicle node, the buffer node, the work node, the source node and the sink node. We expect that nodes support an easy way to model the MHS simulation model.

The proposed simulation program has been tested by using refrigerator assembly line example which is most problematic assembly line in the five electronics assembly lines. We can model MHS simulation model of the refrigerator assembly line with two routes in 20 minutes. After MHS simulation, we confirmed the number of starvation times in each buffers and the congestion level in the MHS. However, there are not criteria to estimate the congestion degree in the assembly line, and, the report of the 
MHS simulation result is not intuitive. Therefore, the congestion criteria and the intuitive report method are recommended to estimate the congestion level of the assembly line. Furthermore, the research which searches and classifies items to determine feasibility of the MHS is recommended.

\section{ACKNOWLEDGMENT}

This work (Grants No.C0003579) was supported by Business for Cooperative R\&D between Industry, Academy, and Research Institute funded Korea Small and Medium Business Administration in 2013.

\section{REFERENCES}

[1] D. G. Conway and M. A. Venkataramanan, "Genetic search and the dynamic facility layout problem," Computers and Operation Research, vol. 21, pp. 955-960, 1994.

[2] R. H. Shore and J. A. Tompkins, "Flexible facilities design," AIIE Transactions, vol. 12, pp. 200-205, 1980.

[3] R. Pandit and U. S. Palekar, "Response time considerations for optimal warehouse layout design," Transactions of the ASME, vol. 115, pp. 314-330, 1993.

[4] U. Smutkupt and S. Wimonkasame, "Plant layout design with simulation," in Proc. the International Multi Conference of Engineers and Computer Scientists, vol. 2, 2009.

[5] D. Sinriech and E. Samakh, "A genetic approach to the pickup/delivery station location problem in segmented flow based material handling systems," Journal of Manufacturing Systems, vol. 18, pp. 81-99, 1999.

[6] J. W. Herrmann, G. loannou, and I. Minis, "Design of material flow networks in manufacturing facilities," Journal of Manufacturing Systems, vol. 14, pp. 278-289, 1995.

[7] G. Ioannou, "An integrated model and a decomposition-based approach for concurrent layout and material handling system design," Computers\&Industrial Engineering, vol. 52, pp. 459-485, 2007.
[8] N. Brauner and G. Finke, "Optimal moves of the material handling system in a robotic cell," International Journal of Production Economics, vol. 74, pp. 269-277, 2001.

[9] L. R. Foulds. "Layout manager: A microcomputer based decision support system for facilities layout," Decision Support Systems, vol. 20, pp. 199-213, 1997.

[10] A. Nozari and E. E. Enscore Jr., "Computerized facility layout with graph theory," Computers \&Idustrial Engineering, vol. 5, pp. 183-193, 1981.

[11] A. Kusiak and S. S. Heragu, "The facility layout problem," European Journal of Operational Research, vol. 27, pp. 229-251, 1987.

[12] G. Evans, M. Wilhelm, and W. Karwowski, "A layout design heuristic employing the theory of fuzzy sets," International Journal of Production Research, vol. 25, pp. 1431-1450, 1987.

[13] D. S. Chang, E. Ahn, and S. C. Park. "Topology based assembly production line layout generation," in Proc. the Society of CAD/CAM Engineers Conference, 2012, pp. 545-549.

[14] T. G. Kim, DEVSIM++ User's Manual, Korea: Department of Electrical Engineering, KAIST, 1994.

[15] B. P. Zeigler, Multifacetted Modeling and Discrete Event Simulation, New York: Academic Press, 1984.

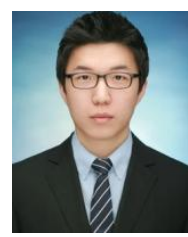

Dae S. Chang received a bachelor degree in 2012 in the Department of Industrial engineering at Ajou University, Korea. He is now a graduate student in industrial engineering, Ajou University, Korea, and he is a member of modeling and simulation laboratory, which is an affiliation of Department of industrial engineering, Ajou University. $\mathrm{He}$ is interested in distributed simulation system, digital manufacturing system, and CAD/CAM/CAPP.

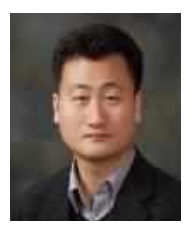

Sang C. Park is a professor in the Department of Industrial Engineering at Ajou University. Before joining Ajou, he worked for DaimlerChrysler Corp. and CubickTek Co., developing commercial and in-house CAD/CAM/CAPP/Simulation software systems. He received his BS, MS and Ph.D. degrees from KAIST in 1994, 1996 and 2000, respectively, all in industrial engineering. His research interests include geomet-ric algorithms in $\mathrm{CAD} / \mathrm{CAM}$, process planning, engineering knowledge management, and discrete event system simulation. 